\title{
Editorial
}

\section{The Epidemiology and Control of VRE: Still Struggling to Come of Age}

\author{
C. Glen Mayhall, MD
}

Enterococci have caused infections in hospitalized patients for many decades. Given that they were part of the normal flora of the gastrointestinal (G) tract and that they frequently appeared as part of the flora in infections related to fecal contamination, they originally were considered endogenous pathogens of little nosocomial import. This view began to change with the appearance of enterococci resistant to vancomycin in Europe in $1988 .{ }^{1}$ Vancomycin resistance appeared subsequent to earlier reports of enterococci resistant to pencillin ${ }^{2}$ and aminoglycosides, ${ }^{3,4}$ raising the concern that infections caused by vancomycin-resistant enterococci (VRE) might be difficult, if not impossible, to treat.

A 1993 report $^{5}$ from the National Nosocomial Infection Surveillance system at the Centers for Disease Control and Prevention (CDC) indicated that VRE had appeared in the United States and had increased from 0.3\% of nosocomial enterococci in 1989 to $7.9 \%$. The change was even more dramatic for enterococcal isolates in intensive care units, where VRE had increased in the same period from $0.4 \%$ to $13.6 \%$, a 34 -fold increase in the 4-year period.

During the period covered by that report, six VRE outbreaks were reported in the literature..$^{6-11}$ In 1994, three more publications described outbreaks of VRE. ${ }^{12-14}$ These studies during the first 5 years of the VRE epidemic (19891994) began to define the epidemiology of VRE colonization and infection and led to the following conclusions:

- VRE colonize or infect very ill and immunocompromised patients (intensive care, oncology, and transplant patients).

- The GI tract is the most important reservoir.

- VRE contaminate patients' environments.

- Environmental contamination is increased when patients have diarrhea.

- VRE may be transmitted by medical instruments.

- Prolonged hospital stay increases the risk for acquiring VRE.
- Exposure to cephalosporins and vancomycin may increase the risk of colonization by VRE.

- Close proximity to a VRE-positive patient and being cared for by a nurse caring for a VRE-positive patient increases the risk for acquisition of VRE.

- Resistance in VRE is transferable.

- Although not frank pathogens, VRE can cause invasive disease associated with morbidity and possibly mortality.

Presently, the rising epidemic of VRE, the limited or absent therapeutic options for treating serious VRE infections, and a concern that the resistance genes in VRE might be transferred to other gram-positive microorganisms such as Staphylococcus aureus led the CDC to publish recommendations from the Hospital Infection Control Practices Advisory Committee for preventing the spread of vancomycin resistance..$^{15}$ These recommendations were developed using data from studies published during the first 5 years of the VRE epidemic. The recommendations provided guidelines for the prudent use of vancomycin, recommended educational programs for hospital staff regarding the epidemiology and control of VRE, defined the role of the hospital microbiology laboratory, and outlined an approach for the prevention and control of VRE. Recommendations for control included culture surveys to identify patients colonized with VRE, barrier precautions, environmental decontamination, and establishment of a mechanism to ensure that VRE carriers were placed on Isolation Precautions immediately on readmission to the hospital. Since publication of these recommendations, VRE have continued to spread, and the epidemic has not been controlled. In the second 5 years of the epidemic (1994 to the present), only modest additional contributions have been made to our understanding of the epidemiology and control of VRE.

Compared to the case-control studies published between 1989 and 1994, case-control studies published in 
the last 5 years were more likely to be analyzed with multivariable techniques ${ }^{16-19}$ but were no more likely to determine clearly the time of acquisition of VRE, making it impossible to ascertain whether risk-factor data were collected before occurrence of this outcome. One of the articles in this issue of Infection Control and Hospital Epidemiology, while otherwise well-designed and analyzed, fails to identify the time of onset of the outcome enteric colonization $^{20}$; thus, it is likely that some risk-factor data were collected after the onset of colonization. Since the onset of colonization is a "silent" event, determining the time of onset of colonization may be challenging, particularly for retrospective studies.

Studies published in the last 5 years that have determined the onset of colonization accurately have confirmed cephalosporin use and length of stay to be risk factors for VRE and have added enteric feeding, proportion of days on enteric feeding, use of sucralfate, and "colonization pressure" as additional risk factors for acquisition of VRE. ${ }^{17,19}$

Environmental contamination with VRE also has been a subject of study in the last 5 years. Two studies have shown that VRE can remain viable on inanimate surfaces for 7 days to 2 months. ${ }^{21,22}$ In a prospective study of patients admitted to a medical intensive care unit, Bonten and coworkers observed contamination in $63 \%$ of the rooms of patients on mechanical ventilation ${ }^{23}$ and noted that environmental contamination was more likely to occur in rooms of patients with a higher proportion of body sites colonized with VRE. They found that environmental contamination was more transient than patient colonization and that only $3(23 \%)$ of 13 patients culture-negative for VRE became culture-positive after they were cared for in rooms with environmental contamination. Since the authors did not use broth enrichment for environmental cultures, it is unclear whether apparent transient environmental colonization and the low colonization rate for patients exposed to environments culture-positive for VRE might have been due to failure to identify all contaminated environmental surfaces. Ford and colleagues observed only $42 \%$ of isolates identified by broth enrichment were identified by direct plating of swabs on solid media. ${ }^{24}$ We have found an even lower recovery rate for direct plating of swabs used to sample environmental surfaces when compared to broth enrichment (B.S. Reisner, PhD, unpublished data, August 1999).

Smith and associates, using a broth enrichment culture technique, showed that environmental contamination may occur in the outpatient setting. ${ }^{25}$ However, these investigators sampled only seven rooms, and only two sites in each of two rooms were positive. These interesting observations should lead to larger studies with many sites sampled, and sites of colonization in patients should be correlated with the sites and degree of environmental contamination.

Additional studies have also been published on the role of healthcare workers (HCWs) in the transmission of VRE by contaminated hands or as possible reservoirs for VRE. As in the first 5 years of the epidemic, ${ }^{14}$ most investigators have not succeeded in recovering VRE from the hands of $\mathrm{HCWs}^{16}$ or have recovered VRE on culture of hands from a very small percentage of HCWs. ${ }^{26}$ Unlike the findings of the latter studies, Bonilla and colleagues recovered VRE from the hands of $13 \%$ to $41 \%$ of HCWs over 3 years in a long-term-care unit. ${ }^{27}$ In a mathematical model of transmission dynamics, persistence, and the impact of infection control programs on VRE in an intensive care unit setting, Austin and colleagues showed that the prevalence of contamination of HCWs' hands was very low, even though the incidence may have been high, and offered this observation as an explanation for the low recovery of VRE from cultures of HCWs' hands. ${ }^{28}$

Studies of GI colonization of HCWs with VRE have shown that such colonization occurs uncommonly. In a study published in 1993, $4(6.5 \%)$ of $62 \mathrm{HCWs}$ had rectal swabs positive for VRE. ${ }^{11}$ In three studies, VRE were recovered from rectal cultures of 1 of $27,{ }^{23}$ none of $34,,^{14}$ and none of $55^{29} \mathrm{HCWs}$. The latter survey was performed during a nonepidemic period with an estimated prevalence of VRE of $2.1 \%$. It would appear that $\mathrm{HCWs}$ are not an important reservoir for VRE.

Austin and colleagues' mathematical model identified hand washing and cohorting as important control measures and showed how restriction of antibiotic use could substantially lower the transmission of VRE. ${ }^{28}$ The authors showed that the model predicted the observed pattern with good agreement qualitatively and quantitatively. Development of this model is a significant advance in understanding the epidemiology and control of VRE. The model is limited by the assumption by the authors that the environment does not play an important role in the epidemiology of VRE. However, most studies of the environment have not used techniques sufficiently sensitive to detect low levels of surface contamination. This is another example of the need for better studies of environmental contamination by VRE. Another factor that mathematical models may need to take into account is the condition of the exposed patient's GI tract. Do changes brought about by the patient's underlying illness, changes in GI tract function, and oral and systemic medications affect the likelihood that the patient will develop colonization after exposure?

In summary, with a few notable exceptions, not much progress has been made in understanding the epidemiology and in identifying effective methods for controlling VRE in healthcare settings in the last 5 years. There have been few cohort or case-control studies; some have not been well designed, and all have been very limited in the scope of potential risk factors examined. Larger studies are needed that sharply define the onset of the outcome and that examine a larger number of potential risk factors, taking into account the potential modes of transmission and inoculation of the host with VRE, as well as factors that might decrease or increase the likelihood that a given VRE inoculum will cause colonization of the host. The effects of the physiological state of the GI tract and of medications other than antibiotics on colonization of the GI tract should be studied. The effects of sedative and narcotic drugs, laxatives, medications given for the symptomatic relief of diarrhea, $\mathrm{H}_{2}$ blockers, and antacids should be examined. 
Better studies are needed of the role played by environmental contamination in the epidemiology of VRE. Persistence of VRE in the environment is well established. However, it remains unclear how important the environment is as a source of VRE for colonization of patients. What sites in the environment are most likely to be sources of VRE for patients? Is the density of contamination on environmental surfaces related to risk of acquisition? What are the modes of transmission of VRE from environmental surfaces to patients? Are risk factors for acquisition of VRE from the environment different from risk factors for acquisition of VRE from other patients? Large prospective studies designed to answer these questions will be needed if we are to understand the role of the environment in the epidemiology of VRE. These studies should be carried out in acute-care settings, long-term-care facilities, and outpatient clinics. Only then will we be able to understand whether we need to develop control measures based on environmental contamination, and what they should be.

Healthcare workers do not seem to be important reservoirs for VRE, and it would appear that HCWs infrequently transmit VRE between patients on their hands. To transmit VRE between patients by hand contact requires that HCWs fail to don gloves before entering a VRE patient's room, fail to wash hands after leaving the room, and fail to don gloves before entering a susceptible patient's room. This may explain the uncommon recovery of VRE from the ungloved hands of HCWs in most studies. On the other hand, it is unknown how often HCWs transmit VRE to patients on their gloved hands from sites of environmental contamination after entering a patient's room.

Substantial progress in our understanding of the epidemiology and control of VRE in the next 5 years will depend on commitment of sufficient resources to carry out larger, prospective, well-designed studies to define all of the important risk factors for acquisition of VRE and to understand fully the importance of the environment in the epidemiology of VRE.

\section{REFERENCES}

1. Uttley AH, Collins $\mathrm{CH}$, Naidoo J, George RC. Vancomycin-resistant enterococci. Lancet 1988;1:57-58.

2. Bush LM, Calmon J, Cherney CL, Wendeler M, Pitsakis P, Poupard J, et al. High-level penicillin resistance among isolates of enterococci. Implications for treatment of enterococcal infections. Ann Intern Med 1989;110:515-520.

3. Zervos MJ, Kauffman CA, Therasse PM, Bergman AG, Mikesell TS, Schaberg DR. Nosocomial infection by gentamicin-resistant Streptococcus faecalis. An epidemiologic study. Ann Intern Med 1987;106:687-691.

4. Noskin GA, Till M, Patterson BK, Clarke JT, Warren JR. High-level gentamicin resistance in Enterococcus faecalis bacteremia. I Infect Dis 1991;164:1212-1215.

5. Centers for Disease Control and Prevention. Nosocomial enterococci resistant to vancomycin-United States, 1989-1993. MMWR 1993;42: 597-599.

6. Uttley AH, George RC, Naidoo J, Woodford N, Johnson AP, Collins CH, et al. High-level vancomycin-resistant enterococci causing hospital infections. Epidemiol Infect 1989;103:173-181.

7. Karanfil LV, Murphy M, Josephson A, Gaynes R, Mandel L, Hill BC, et al. A cluster of vancomycin-resistant Enterococcus faecium in an intensive care unit. Infect Control Hosp Epidemiol 1992;13:195-200.

8. Livornese LL Jr, Dias S, Samel C, Romanowski B, Taylor S, May P, et al.
Hospital-acquired infection with vancomycin-resistant Enterococcus faecium transmitted by electronic thermometers. Ann Intern Med 1992;117:112-116.

9. Rubin LG, Tucci V, Cercenado E, Eliopoulos G, Isenberg HD. Vancomycin-resistant Enterococcus faecium in hospitalized children. Infect Control Hosp Epidemiol 1992;13:700-705.

10. Boyle JF, Soumakis SA, Rendo A, Herrington JA, Gianarkis DG, Thurberg BE, et al. Epidemiologic analysis and genotypic characterization of a nosocomial outbreak of vancomycin-resistant enterococci. $J$ Clin Microbiol 1993;31:1280-1285.

11. Handwerger S, Raucher B, Altarac D, Monka J, Marchione S, Singh $\mathrm{KV}$, et al. Nosocomial outbreak due to Enterococcus faecium highly resistant to vancomycin, penicillin, and gentamicin. Clin Infect Dis 1993:16:750-755.

12. Boyce JM, Opal SM, Chow JW, Zervos MJ, Potter-Bynoe G, Sherman $\mathrm{CB}$, et al. Outbreak of multidrug-resistant Enterococcus faecium with transferable van $\mathrm{B}$ class vancomycin resistance. J Clin Microbiol 1994;32:1148-1153.

13. Montecalvo MA, Horowitz H, Gedris C, Carbonaro C, Tenover FC, Issah A, et al. Outbreak of vancomycin-, ampicillin-, and aminoglycoside-resistant Enterococcus faecium bacteremia in an adult oncology unit. Antimicrob Agents Chemother 1994;38:1363-1367.

14. Jordens JZ, Bates J, Griffiths DT. Faecal carriage and nosocomial spread of vancomycin-resistant Enterococcus faecium. J Antimicrob Chemother 1994;34:515-528.

15. Hospital Infection Control Practices Advisory Committee. Recommendations for preventing the spread of vancomycin resistance. Infect Control Hosp Epidemiol 1995; 16:105-113.

16. Morris JG Jr, Shay DK, Hebden JN, McCarter RJ Jr, Perdue BE, Jarvis $\mathrm{W}$, et al. Enterococci resistant to multiple antimicrobial agents, including vancomycin. Establishment of endemicity in a university medical center. Ann Intern Med 1995;123:250-259.

17. Slaughter S, Hayden MK, Nathan C, Hu TC, Rice T, van Voorhis J, et al. A comparison of the effect of universal use of gloves and gowns with that of glove use alone on acquisition of vancomycin-resistant enterococci in a medical intensive care unit. Ann Intern Med 1996;125:448-456.

18. Tornieporth NG, Roberts RB, John J, Hafner A, Riley LW. Risk factors associated with vancomycin-resistant Enterococcus faecium infection or colonization in 145 matched case patients and control patients. Clin Infect Dis 1996;23:767-772.

19. Bonten MJ, Slaughter S, Ambergen AW, Hayden MK, van Voorhis J, Nathan C, et al. The role of "colonization pressure" in the spread of vancomycin-resistant enterococci. An important infection control variable. Arch Intern Med 1998;158:1127-1132.

20. Garbutt JM, Littenberg B, Evanoff BA, Sahm D, Mundy LM. Enteric carriage of vancomycin-resistant Enterococcus faecium in patients tested for Clostridium difficile. Infect Control Hosp Epidemiol 1999;20:664-670.

21. Noskin GA, Stosor V, Cooper I, Peterson LR. Recovery of vancomycinresistant enterococci on fingertips and environmental surfaces. Infect Control Hosp Epidemiol 1995;16:577-581.

22. Bonilla HF, Zervos MJ, Kauffman CA. Long-term survival of vancomycin-resistant Enterococcus faecium on a contaminated surface. Infect Control Hosp Epidemiol 1996;17:770-772.

23. Bonten MJ, Hayden MK, Nathan C, van Voorhis J, Matushek M, Slaughter S, et al. Epidemiology of colonisation of patients and environment with vancomycin-resistant enterococci. Lancet 1996;348:1615-1619.

24. Ford M, Perry JD, Gould FK, Orr KE. Neomycin blood agar as a selective medium for vancomycin resistant Enterococcus faecium. J Clin Pathol 1996;49:437.

25. Smith TL, Iwen PC, Olson SB, Rupp ME. Environmental contamination with vancomycin-resistant enterococci in an outpatient setting. Infect Control Hosp Epidemiol 1998;19:515-518.

26. Wells CL, Juni BA, Cameron SB, Mason KR, Dunn DL, Ferrieri P, et al. Stool carriage, clinical isolation, and mortality during an outbreak of vancomycin-resistant enterococci in hospitalized medical and/or surgical patients. Clin Infect Dis 1995;21:45-50.

27. Bonilla HF, Zervos MA, Lyons MJ, Bradley SF, Hedderwick SA, Ramsey MA, et al. Colonization with vancomycin-resistant Enterococcus faecium: comparison of a long-term-care unit with an acute-care hospital. Infect Control Hosp Epidemiol 1997;18:333-339.

28. Austin DJ, Bonten MJ, Weinstein RA, Slaughter S, Anderson RM. Vancomycin-resistant enterococci in intensive-care hospital settings: transmission dynamics, persistence, and the impact of infection control programs. Proc Natl Acad Sci USA 1999;96:6908-6913.

29. Carmeli Y, Venkataraman L, DeGirolami PC, Lichtenberg DA, Karchmer AW, Samore MH. Stool colonization of healthcare workers with selected resistant bacteria. Infect Control Hosp Epidemiol 1998;19:38-40. 